\title{
EXISTENCE AND MULTIPLICITY OF POSITIVE RADIAL SOLUTIONS FOR SEMILINEAR ELLIPTIC EQUATIONS IN ANNULAR DOMAINS*
}

\author{
SONG-SUN LIN†‡ AND FENG-MING PAI $\uparrow$
}

\begin{abstract}
The existence and multiplicity of positive radial solutions of equation $\Delta u+f(u)=0$ is studied in annular domains in $\mathbb{R}^{n}, n \geqq 2$. It is proven that if $f(0) \geqq 0, f$ is somewhere negative in $(0, \infty)$ and superlinear at $\infty$, then there is a large positive radial solutions on all annuli. If $f(0)<0$ and satisfies certain conditions, then the equation has no solution if the annuli are too wide. Multiplicity results are also obtained when $f$ has many humps with positive areas.
\end{abstract}

Key words. elliptic, semilinear, positive radial solution, annular domain

AMS(MOS) subject classifications. 35B32, 35JB65, 35P30

1. Introduction. In this paper we consider the existence and multiplicity of positive radial solutions of the semilinear elliptic equation

$$
\begin{aligned}
& \Delta u(x)+f(u(x))=0 \quad \text { in } a<|x|<b, \\
& u(x)=0 \quad \text { on }|x|=a \text { and }|x|=b,
\end{aligned}
$$

$x \in \mathbb{R}^{n}, n \geqq 2$ and $f \in C^{1}((0, \infty)) \cap C^{0}([0, \infty))$ satisfying the following hypotheses:

(H1) $\quad f$ is negative somewhere in $(0, \infty)$;

(H2) $f$ is superlinear at $u=\infty$, i.e., $\lim _{u \rightarrow \infty} f(u) / u=\infty$.

One of the problems for semilinear elliptic equations in annular domains which have been studied quite extensively in recent years is:

(P) Does (1.1), (1.2) possess a positive radial solution in every annulus?

The answer to (P) was proved affirmative by Nehari [20], assuming that $f$ is positive in $(0, \infty)$ and satisfies the condition: $\exists \delta>0$ such that $f(u) / u^{1+\delta}$ is monotone increasing in $(0, \infty)$.

Later, (P) was studied by Kazdan and Warner [15], Ni and Nussbaum [21], Bandle, Coffman, and Marcus [2], Garaizar [13], and Lin [17].

In [2], Bandle, Coffman, and Marcus showed that the answer to $(\mathrm{P})$ is affirmative, provided that $f$ is positive in $(0, \infty)$ and satisfies the following conditions:

(A1) $\quad f$ is nondecreasing in $(0, \infty)$;

(A2) $\quad \lim _{u \rightarrow 0} f(u) / u=0$;

(A3) $\quad \lim _{u \rightarrow \infty} f(u) / u=\infty$.

In [2], it is remarked that (A1) is not a necessary condition for existence. This have been confirmed by Coffman and Marcus [8] and Lin [17] independently.

With a suitable change of independent variable, (1.1), (1.2) become equations of the form

$$
\begin{aligned}
& u^{\prime \prime}(t)+G(t, u)=0, \quad t_{0}<t<t_{1}, \\
& u\left(t_{0}\right)=0=u\left(t_{1}\right)
\end{aligned}
$$

\footnotetext{
* Received by the editors January 22, 1990; accepted for publication January 7, 1991.

$\dagger$ Department of Applied Mathematics, National Chiao Tung University, Hsin-chu, Taiwan, Republic of China. China.

$\ddagger$ The work of this author was partially supported by the National Science Council of the Republic of
} 
In [3], Bandle and Kwong showed that the answer to (P) is affirmative if $G$ satisfies the following conditions:

(G1) $\quad G$ is $C^{0}$ in its first variable and $C^{1}$ in its second;

(G2) $\lim _{u \rightarrow \infty} G(t, u) / u=\infty$ uniformly on every $\left[t_{0}, t_{1}\right]$;

(G3) $\lim _{u \rightarrow 0} G(t, u) / u=0$ uniformly on every $\left[t_{0}, t_{1}\right]$.

$G(t, u)$ is now allowed to be negative for small positive value and $G(t, 0)=0$ is assumed implicitly with the limit involved exists and finite in (G3).

In this paper, we first generalize the results of Bandle and Kwong [3], showing that $(\mathrm{P})$ is affirmative if $(\mathrm{H} 1),(\mathrm{H} 2)$, and $(\mathrm{H} 3)$ are satisfied.

$$
f(0) \geqq 0 .
$$

Moreover, solutions obtained are "large" in the following sense: By (H1), there exists $\left(u_{*}, u^{*}\right) \subset(0, \infty)$ such that

$$
f(u) \geqq 0 \quad \text { in }\left(u^{*}, \infty\right), \quad f(u)<0 \quad \text { in }\left(u_{*}, u^{*}\right), \quad f\left(u_{*}\right)=f\left(u^{*}\right)=0 .
$$

Let $\gamma>u^{*}$ be the smallest number such that

$$
\int_{u_{*}}^{\gamma} f(u) d u=0 .
$$

The solution $u$ of $(1.1),(1.2)$ is called large if

$$
\|u\| \equiv \max \{u(x): a \leqq|x| \leqq b\} \geqq \gamma .
$$

On the contrary, Garaizar [13] showed that (P) is negative, i.e., (1.1), (1.2) has no positive radial solution if $b-a$ is too large, if $f$ satisfies the following conditions:

(i) $f(0)<0$;

(ii) There exists $\bar{u}>0$ such that $F(u) \leqq 0$ in $(0, \bar{u})$ and $f(u)>0$ in $(\bar{u}, \infty)$;

(iii) There exists $k>1$ and $d_{2} \geqq d_{1}>0$ such that $d_{1} u^{k} \leqq f(u) \leqq d_{2} u^{k}$ for $u$ large, where

$$
F(u)=\int_{0}^{u} f(t) d t
$$

We can also obtain a similar nonexistence result without assuming condition (iii), i.e., if ( $\mathrm{H} 2)$ and the following hold true:

(H3)'(i) $f(0)<0$;

(ii) There exists $\bar{u}>0$ such that $F(u)<0$ in $(0, \bar{u}]$ and $f(u)>0$ in $(\bar{u}, \infty)$.

On the other hand, when $f$ changes signs, the existence of multiple positive solutions of the equation

$$
\begin{aligned}
& \Delta u+\lambda f(u)=0 \text { in } \Omega, \\
& u=0 \text { on } \partial \Omega,
\end{aligned}
$$

$\lambda \geqq 0$ and $\Omega$ is a bounded smooth domain in $\mathbb{R}^{n}, n \geqq 2$, has been studied by many authors (see, e.g., Brown and Budin [5], Hess [14], de Figueiredo [12], Clement and Sweers [6], and Wang and Kazarinoff [24]).

In [14], Hess showed that if $f$ satisfies the following conditions:

(f $f(0)>0$;

$\left(\mathrm{f}_{2}\right)$ There exist $m$ numbers $\bar{u}_{m}>\bar{u}_{m-1}>\cdots>\bar{u}_{1}>0$ such that $f\left(\bar{u}_{k}\right)=0$ for $k=$ $1, \cdots, m$;

$\left(\mathrm{f}_{3}\right) 0<\max \left\{F(s): 0 \leqq s \leqq \bar{u}_{k-1}\right\}<F\left(\bar{u}_{k}\right), k=2, \cdots, m$,

then there exists a number $\bar{\lambda}>0$ such that for all $\lambda>\bar{\lambda},(1.7),(1.8)$ have at least $2 m-1$ positive solutions $\hat{u}_{1}, u_{2}, \hat{u}_{2}, \cdots, u_{m}, \hat{u}_{m}$ such that $\left\|\hat{u}_{1}\right\|<\bar{u}_{1}$ and $\bar{u}_{k-1}<\left\|u_{k}\right\|,\left\|\hat{u}_{k}\right\|<\bar{u}_{k}$ for $k=2, \cdots, m$, and $\hat{u}_{k-1}<\hat{u}_{k}$ and $u_{k}<\hat{u}_{k}$ for $k=2, \cdots, m$. 
Later, de Figueiredo [12] obtained the existence of $2 m-1$ ordered positive solutions under slightly different assumptions.

In this paper, we show that if $f$ satisfies $\left(\mathrm{f}_{2}\right)$ and $\left(\mathrm{f}_{3}\right)$, then there exists $b_{m}>a$ such that for any $b>b_{m},(1.1),(1.2)$ has at least $m$ ordered positive radial solutions $u_{k}$ with $\left\|u_{k}\right\| \in\left(\bar{u}_{k-1}, \bar{u}_{k}\right)$ for $k=2, \cdots, m$. Moreover, if $f(0) \geqq 0$, then (1.1), (1.2) has at least $2 m-1$ positive radial solutions for $b>b_{m}$.

For the other related problems, note the following:

(i) Uniqueness of positive radial solution, when $f(u)>0$ for $u \in(0, \infty)$, has been studied by $\mathrm{Ni}$ and Nussbaum [21], Bandle, Coffman, and Marcus [2], Bandle and Kwong [3], and Coffman and Marcus [8].

(ii) Symmetry breaking for positive radial solutions has been studied by Brezis and Nirenberg [4], Coffman [7], Suzuki and Nagasaki [22], [23], and Lin [16], [18], [19].

The methods used in this paper are shooting techniques, the phase-plane method, and variational methods. All results obtained in this paper can also be generalized to $f(r, u)$ which satisfies certain uniformity assumptions in $r$ as in (G2) and (G3).

The paper is organized as follows. In $\S 2$, we obtain some preliminary results which are useful. In $\S 3$, we prove that $(\mathrm{P})$ is affirmative when $(\mathrm{H} 1) \sim(\mathrm{H} 3)$ are satisfied. In $\S 4$, we prove $(\mathrm{P})$ is negative when $(\mathrm{H} 1) \sim(\mathrm{H} 3)^{\prime}$ are satisfied. In $\S 5$, we obtain the multiplicity results for wide annuli.

2. Preliminaries. Since we are interested in positive radial solutions of (1.1), we write (1.1), (1.2) in the form

$$
\begin{gathered}
u^{\prime \prime}(r)+\frac{n-1}{r} u^{\prime}(r)+f(u(r))=0 \quad \text { in }(a, b), \\
u(a)=0=u(b) .
\end{gathered}
$$

For fixed $a>0$, we consider the family of solutions $u(\cdot) \equiv u(\cdot, \alpha)$ of the initial value problem

$$
\begin{gathered}
u^{\prime \prime}(r)+\frac{n-1}{r} u^{\prime}(r)+f(u(r))=0 \quad \text { for } r>a, \\
u(a)=0 \quad \text { and } \quad u^{\prime}(a)=\alpha,
\end{gathered}
$$

where $\alpha \geqq 0$ is the shooting parameter. Furthermore, (2.3), (2.4) can also be written as a dynamical system

$$
\begin{gathered}
u^{\prime}=v, \\
v^{\prime}=-\frac{n-1}{r} v-f(u),
\end{gathered}
$$

with initial data

$$
u(a)=0 \quad \text { and } \quad v(a)=\alpha .
$$

We define an energy function $H(\cdot) \equiv H(u(\cdot, \alpha))$ by

$$
H(r)=\frac{1}{2} v^{2}(r)+F(u(r)) .
$$

Then, along each trajectory of solution of (2.5), (2.6), $H$ is decreasing; in fact,

$$
H^{\prime}(r)=-\frac{n-1}{r} u^{\prime 2}(r) \leqq 0 .
$$

Furthermore, $H(r)$ is strictly decreasing in $r$ if $\alpha>0$. 
We first classify the solutions $u(\cdot, \alpha)$.

Definition 2.1. For any $\alpha \geqq 0, \alpha$ belongs to one of the following three disjoint sets:

(i) $\alpha \in P$ (or $u(\cdot, \alpha)$ is a $P$-solution) if $u(r, \alpha)>0$ for all $r>a$,

(ii) $\alpha \in N$ (or $u(\cdot, \alpha)$ is an $N$-solution) if there exists $b(\alpha)>0$ such that $u(r, \alpha)>$ 0 in $(a, b(\alpha)), u(b(\alpha), \alpha)=0$ and $u^{\prime}(b(\alpha), \alpha)<0$,

(iii) $\alpha \in T$ (or $u(\cdot, \alpha)$ is a $T$-solution) if there exists $b(\alpha)>0$ such that $u(r, \alpha)>0$ in $(a, b(\alpha)), u(b(\alpha), \alpha)=0$ and $u^{\prime}(b(\alpha), \alpha)=0$.

We then state some simple but basic properties of solutions $u(\cdot, \alpha)$.

LEMMA 2.2. (i) If $\alpha \in N$, then $u(\cdot, \alpha)$ has only one local maximum.

(ii) If $\alpha \in N \cup T$, then $H(u(r, \alpha))>0$ for $r \in(a, b(\alpha))$.

(iii) If ( $\mathrm{H} 2)$ is satisfied and $u(r, \alpha)>0$ for all $r>r_{0} \geqq a$, then $u(r, \alpha)$ is bounded.

(iv) $N$ is an open set.

Proof. (i) The proof of (i), in the general case, was given by Garaizar [13]. The main idea is using energy $H(r)$, which decreases along the trajectory, and then obtain the following two facts:

(a) the trajectory cannot cross (intersect) itself;

(b) the trajectory cannot be tangent to the $u$-axis.

Therefore, (i) can be proved. For the details, see [13, Lemma 1].

(ii) Since $H(u(b(\alpha), \alpha)) \geqq 0$, (ii) follows.

(iii) Since

$$
H(u(r, \alpha))=\frac{1}{2} u^{\prime 2}(r)+F(u(r, \alpha)) \leqq H(u(a, \alpha))=\alpha^{2} / 2,
$$

we have $F(u(r, \alpha)) \leqq \alpha^{2} / 2$ for all $r \geqq r_{0}$. Therefore, (H2) implies $u(r, \alpha)$ is bounded.

(iv) By the Implicit Function Theorem, $b(\alpha)$ is continuously differentiable in $N$ and $N$ is an open set.

The following lemma indicates there is a great difference between cases $f(0) \geqq 0$ and $f(0)<0$.

LemmA 2.3. If $f(0) \geqq 0$, then $T=\phi$. Furthermore, if $\alpha \in T$ then $u(r, \alpha)>0$ for all $r>b(\alpha)$.

Proof. If $f(0)=0$, then $(u, v)=(0,0)$ is an equilibrium. Hence, $T=\phi$. If $f(0)>0$ and there were $\alpha \in T$, then $u^{\prime \prime}(b(\alpha), \alpha)=-f(0)<0$. Therefore, $u(r, \alpha)<0$ for $r<b(\alpha)$ and sufficiently close to it, a contradiction. This proves $T=\phi$.

If $\alpha \in T$, then it is necessary that $f(0)<0$. Since $H(u(b(\alpha), \alpha))=0$ implies that $H(u(r, \alpha))<0$ for all $r>b(\alpha)$, then $u^{\prime \prime}(b(\alpha), \alpha)=-f(0)>0$ implies that $u(r, \alpha)>0$ for all $r>b(\alpha)$.

The following lemma plays a crucial role in the study of problem (P).

LEMMA 2.4. If there is a sequence $\left\{\alpha_{k}\right\} \subset N \cup T$ such that

$$
\alpha_{k} \rightarrow \bar{\alpha}>0 \text { and } b\left(\alpha_{k}\right) \rightarrow \infty
$$

as $k \rightarrow \infty$, then $\bar{\alpha} \in P$ and $u(\cdot, \bar{\alpha})$ satisfies the following monotonicity property:

(M) (i) $u(r, \bar{\alpha})$ is either strictly increasing in $(a, \infty)$ or there exists $a_{1}>a$ such that $u(r, \bar{\alpha})$ is strictly increasing in $\left(a, a_{1}\right)$ and strictly decreasing in $\left(a_{1}, \infty\right)$.

(ii) $u(r, \bar{\alpha}) \rightarrow \tilde{u}$ as $r \rightarrow \infty$ where $f(\tilde{u})=0$.

Proof. First, we observe that $u(\cdot, \bar{\alpha})$ cannot have a local maximum followed by a local minimum. Otherwise, by continuous dependence of ordinary differential equations (o.d.e.), for $k$ sufficiently large, $u\left(r, \alpha_{k}\right)$ will have at least two local maxima in $\left(a, b\left(\alpha_{k}\right)\right)$, a contradiction to Lemma 2.2(i). It is also clear that $u(r, \bar{\alpha})$ cannot be constant on any finite interval of $(a, \infty)$. Hence, $u(\cdot, \bar{\alpha})$ satisfies $(\mathbf{M})(\mathrm{i})$. Condition (M)(ii) follows by Lemma 2.6 which will be proved later. 
As in [2], [3], and [17], it is sometimes convenient to study the existence problem in the form of (1.3), (1.4).

For $n \geqq 3$, in terms of variables

$$
s=r^{2-n} \quad \text { and } \quad w(s)=u(r),
$$

equations (2.1), (2.2) can be rewritten as

$$
\begin{aligned}
& w^{\prime \prime}(s)+\rho(s) f(w(s))=0 \quad \text { in }\left(s_{0}, s_{1}\right), \\
& w\left(s_{0}\right)=0=w\left(s_{1}\right),
\end{aligned}
$$

where $\rho(s)=(n-2)^{-2} s^{-k}, k=(2 n-2) /(n-2), s_{0}=b^{2-n}$, and $s_{1}=a^{2-n}$. For $n=2$, in terms of variables

$$
s=\frac{1}{2}-\log a+\log r \quad \text { and } \quad w(s)=u(r),
$$

equation (2.1) can also be written as (2.11) with $\rho(s)=a^{2} e^{2 s-1}, s_{0}=\frac{1}{2}$ and $s_{1}=$ $-\frac{1}{2}-\log a+\log b$. In the remaining part of the section, we only treat the case $n \geqq 3$; the case $n=2$ can also be treated analogously.

The associated initial value problem, now backward shooting in an $s$-variable, is

$$
\begin{aligned}
& w^{\prime \prime}(s)+\rho(s) f(w(s))=0 \quad \text { for } s<s_{1}, \\
& w\left(s_{1}\right)=0 \quad \text { and } \quad w^{\prime}\left(s_{1}\right)=-\beta,
\end{aligned}
$$

where $\beta \geqq 0$ is the shooting parameter and $s_{1}=a^{2-n}$ is a fixed number.

It is easy to check that $(2.13),(2.14)$ is equivalent to

$$
w(s)=\beta\left(s_{1}-s\right)-\int_{s}^{s_{1}}(t-s) \rho(t) f(w(t)) d t \text { for } s<s_{1},
$$

and the solution $w(\cdot, \beta)$ also satisfies the following equation:

$$
w(s)=w(\bar{s})+w^{\prime}(\bar{s})(s-\bar{s})+\int_{\bar{s}}^{s}(t-s) \rho(t) f(w(t)) d t \quad \text { for } 0<s, \bar{s}<s_{1} .
$$

The associated energy function $V$ is defined by

$$
V(s) \equiv V(w(s, \beta))=\frac{1}{2} w^{\prime 2}(s)+\rho(s) F(w(s)) .
$$

It is clear that

$$
V^{\prime}(s)=\rho^{\prime}(s) F(w(s)),
$$

and so

$$
V(s)=V(\bar{s})+\int_{\bar{s}}^{s} \rho^{\prime}(t) F(w(t)) d t
$$

for $0<s, \bar{s}<s_{1}$.

If $w$ has a zero in $\left(0, s_{1}\right)$, denote

$$
s_{0}(\beta)=\inf \left\{s_{0}: w(s, \beta)>0 \text { in }\left(s_{0}, s_{1}\right)\right\},
$$

and $\nu(\beta) \in\left(s_{0}(\beta), s_{1}\right)$ satisfies

$$
w(\nu(\beta), \beta)=\max \left\{w(s, \beta): s \in\left(s_{0}(\beta), s_{1}\right)\right\} .
$$

With a modification of the argument used in Lin [17], we can prove that $s_{0}(\beta)$ and $\nu(\beta)$ are well defined for sufficiently large $\beta$ and tend to $s_{1}$ as $\beta \rightarrow \infty$. For completeness, we also give a full proof here. 
LEMMA 2.5. If condition $(\mathrm{H} 2)$ is satisfied, then $s_{0}(\beta)$ and $\nu(\beta)$ are well defined for sufficiently large $\beta$. Moreover,

$$
\begin{aligned}
& \lim _{\beta \rightarrow \infty} \nu(\beta)=s_{1}, \\
& \lim _{\beta \rightarrow \infty} s_{0}(\beta)=s_{1},
\end{aligned}
$$

and

$$
\lim _{\beta \rightarrow \infty} w(\nu(\beta), \beta)=\infty .
$$

Proof. We first prove (2.19). If (2.19) were false, then there would be a point $\nu_{0} \in\left(0, s_{1}\right)$ and a sequence $\beta_{k} \rightarrow \infty$ with

$$
w_{k}(s)>0 \quad \text { and } \quad w_{k}^{\prime}(s) \leqq 0 \quad \text { in }\left(\nu_{0}, s_{1}\right),
$$

where $w_{k}(s)=w\left(s, \beta_{k}\right)$.

Letting $\bar{s}=\left(\nu_{0}+s_{1}\right) / 2$, we claim that

$$
\limsup _{k \rightarrow \infty} w_{k}(\bar{s})=\infty
$$

Suppose this is not the case; then there exists a constant $M>0$ such that

$$
w_{k}(\bar{s}) \leqq M \text { for all } k \text {. }
$$

Now, by (2.16) and (2.24), we have

$$
w_{k}(\bar{s})=\frac{1}{2} \beta_{k}\left(s_{1}-\nu_{0}\right)-\int_{\bar{s}}^{s}(t-\bar{s}) \rho(t) f\left(w_{k}(t)\right) d t \geqq \frac{1}{2} \beta_{k}\left(s_{1}-\nu_{0}\right)-C,
$$

for some constant $C \geqq 0$. But, by (2.24), this is impossible. Therefore, (2.23) holds.

By choosing a subsequence of $\beta_{k}$ if necessary, we may assume that

$$
\lim _{k \rightarrow \infty} w_{k}(\bar{s})=\infty
$$

Denote

$$
h_{k}(s)=f\left(w_{k}(s)\right) / w_{k}(s)
$$

in $\left(\nu_{0}, \bar{s}\right)$ and

$$
m_{k}=\inf \left\{h_{k}(s): s \in\left[\nu_{0}, \bar{s}\right]\right\}
$$

By (2.25) and (H2),

$$
\lim _{k \rightarrow \infty} m_{k}=\infty
$$

Now $w_{k}^{\prime \prime}(s)+\rho(s) h_{k}(s) w_{k}(s)=0$ in $\left(\nu_{0}, \bar{s}\right)$ with $\rho(s) h_{k}(s) \geqq \rho(\bar{s}) m_{k}$ in $\left(\nu_{0}, \bar{s}\right)$. By $(2.26)$ and the Sturm Comparison Theorem, $w_{k}$ has zeros in $\left(\nu_{0}, \bar{s}\right)$ for sufficiently large $k$. But by (2.22) this is impossible. This proves (2.19).

Next, we prove (2.21). By (2.18), we have

$$
\frac{1}{2} \beta_{k}^{2}=\rho\left(\nu_{k}\right) F\left(u\left(\nu_{k}\right)\right)+\int_{\nu_{k}}^{s_{1}} \rho^{\prime}(t) F\left(w_{k}(t)\right) d t,
$$

where $\nu_{k}=\nu\left(\beta_{k}\right)$, which implies that $F\left(w_{k}\left(\nu_{k}\right)\right) \rightarrow \infty$ as $k \rightarrow \infty$. By (H2), (2.21) follows. 
Finally, we prove (2.20). If (2.20) were false, then there would be a point $s_{0} \in\left(0, s_{1}\right)$ and a sequence $\beta_{k} \rightarrow \infty$ with

$$
w_{k}(s)>0 \quad \text { in }\left(s_{0}, \nu_{k}\right) \text {. }
$$

Denote $\bar{s}=\frac{1}{2}\left(s_{0}+s_{1}\right)$. By (2.19), we may assume that $\bar{s}<\nu_{k}$ for all $k$. We first claim that

$$
\limsup _{k \rightarrow \infty} w_{k}(\bar{s})<\infty .
$$

Let

$$
L_{k}=\min \left\{w_{k}(s): s \in\left[\bar{s}, \nu_{k}\right]\right\}
$$

Then, there exists $L>0$ such that

$$
L_{k} \leqq L \text { for all } k \text {. }
$$

Otherwise, by the Sturm Comparison Theorem again, $w_{k}$ has a zero in $\left(\bar{s}, \nu_{k}\right)$, a contradiction to (2.27).

If $w_{k}(\bar{s})=L_{k}$, then $(2.28)$ holds.

If $w_{k}(\bar{s})>L_{k}$, let $s_{k} \in\left(\bar{s}, \nu_{k}\right)$ such that $w_{k}\left(s_{k}\right)=L_{k}$. Denote $r_{k}=s_{k}^{1 /(2-n)}, \bar{r}=\bar{s}^{1 /(2-n)}$, and $u_{k}=w_{k}$. Then $u_{k}^{\prime}\left(r_{k}\right)=0$, and we have

$$
H\left(u_{k}\left(r_{k}\right)\right)=F\left(L_{k}\right) \geqq H\left(u_{k}(\bar{r})\right) \geqq F\left(u_{k}(\bar{r})\right) .
$$

By (H2) and (2.29),

$$
u_{k}(\bar{r}) \leqq M
$$

for some constant $M>0$. This proves (2.28).

By (H2), there exists $u^{*}>0$ such that $f(u)>0$ for all $u>u^{*}$. Denote

$$
A_{k}=\left\{s \in\left(0, \tau_{k}\right): w_{k}(s) \leqq u^{*}\right\} .
$$

Then by (2.16), we have

$$
\begin{aligned}
w_{k}\left(\nu_{k}\right) & =w_{k}(\bar{s})+w_{k}^{\prime}(\bar{s})\left(\nu_{k}-\bar{s}\right)+\int_{\bar{s}}^{\nu_{k}}\left(t-\nu_{k}\right) \rho(t) f\left(w_{k}(t)\right) d t \\
& \leqq w_{k}(\bar{s})+w_{k}^{\prime}(\bar{s})\left(\nu_{k}-\bar{s}\right)+\int_{A_{k}}\left(t-\nu_{k}\right) \rho(t) f\left(w_{k}(t)\right) d t \\
& \leqq w_{k}(\bar{s})+w_{k}^{\prime}(\bar{s})\left(\nu_{k}-\bar{s}\right)+C
\end{aligned}
$$

for some constant $C \geqq 0$. Hence, by (2.20), we have

$$
\lim _{k \rightarrow \infty} w_{k}^{\prime}(\bar{s})=\infty \text {. }
$$

On the other hand, by (2.16) again, we have

$$
\begin{aligned}
w_{k}\left(s_{0}\right) & =w_{k}(\bar{s})+w_{k}^{\prime}(\bar{s})\left(s_{0}-\bar{s}\right)+\int_{\bar{s}}^{s_{0}}\left(t-s_{0}\right) \rho(t) f\left(w_{k}(t)\right) d t \\
& \leqq w_{k}(\bar{s})-\frac{1}{2} w_{k}^{\prime}(\bar{s})\left(s_{1}-s_{0}\right)-\int_{A_{k}}\left(t-s_{0}\right) \rho(t) f\left(w_{k}(t)\right) d t \\
& \leqq w_{k}(\bar{s})-\frac{1}{2}\left(s_{1}-s_{0}\right) w_{k}^{\prime}(\bar{s})+C_{1}
\end{aligned}
$$


for some constant $C_{1} \geqq 0$. By (2.28) and (2.30), $w_{k}\left(s_{0}\right) \rightarrow-\infty$ as $k \rightarrow \infty$, a contradiction to (2.27). This proves $(2.20)$.

LEMMA 2.6. If $u(r, \alpha)>0$ for $r>r_{0} \geqq a$, then

$$
\liminf _{r \rightarrow \infty}|u(r, \alpha)-Z|=0,
$$

where $Z=\{\tilde{u} \geqq 0: f(\tilde{u})=0\} \quad$ and $\quad|u-Z|=\inf \{|u-\tilde{u}|: \tilde{u} \in Z\} . \quad$ In particular, if $\lim _{r \rightarrow \infty} u(r, \alpha)=\tilde{u} \geqq 0$, then $f(\tilde{u})=0$.

Proof. If (2.31) were false, then there would be an $\varepsilon>0$ such that

$$
|f(u(r, \alpha))| \geqq \varepsilon .
$$

Denote $w(s, \beta)=u(r, \alpha)$. Then by (2.15)

$$
|w(s, \beta)| \rightarrow \infty \quad \text { as } s \rightarrow 0^{+} \text {. }
$$

This is impossible in viewing $w(s, \beta)>0$ and Lemma 2.2(iii).

3. Existence of large solutions when $f(0) \geqq 0$. In this section we shall prove that if $(H 1) \sim(H 3)$ are satisfied, the answer to $(P)$ is affirmative. We first prove the following lemma.

LEMMA 3.1. If $\alpha \in N$ and $\|u\| \geqq \gamma$ then

$$
\|u\|>\gamma,
$$

where $\gamma$ is in (1.6). A similar result holds for $\alpha \in T$ with

$$
\max \{u(r, \alpha): r \in[a, b(\alpha)]\} \geqq \gamma .
$$

Proof. If $\alpha \in N$, by Lemma 2.2(i), there exists a unique $\tau(\alpha) \in(a, b(\alpha))$, such that

$$
u(\tau(\alpha), \alpha)=\|u\| \text {. }
$$

Let $r_{1}(\alpha) \in(\tau(\alpha), b(\alpha))$ such that

$$
u\left(r_{1}(\alpha), \alpha\right)=u_{*},
$$

where $u_{*}$ is in (1.5). Then

$$
H(u(\tau(\alpha)))=F(u(\tau(\alpha)))>H\left(u\left(r_{1}(\alpha)\right)\right)>F\left(u_{*}\right),
$$

which implies

$$
\int_{u_{*}}^{u(\tau(\alpha))} f(u) d u>0 .
$$

Hence $u(\tau(\alpha))>\gamma$. This proves (3.1). By the same argument, we can obtain (3.1) if $\alpha \in T$ and (3.2) holds.

Lemma 3.2. Assume conditions $(\mathrm{H} 1) \sim(\mathrm{H} 3)$ are satisfied. Then

$$
N_{1}=\{\alpha \in N:\|u(\cdot, \alpha)\|>\gamma\}
$$

is a nonempty open set.

Proof. By Lemmas 2.3 and 2.5, $N_{1}$ is nonempty. By Lemma 3.1 and continuous dependence of o.d.e., $N_{1}$ is an open set.

We can now prove the main result of this section.

TheOREM 3.3. Assume conditions $(\mathrm{H} 1) \sim(\mathrm{H} 3)$ are satisfied. Then for any $b>a>0$, there exists a positive radial solution $u(r)$ of (2.1), (2.2) with $\|u\|>\gamma$.

Proof. By Lemma 3.2, there exists $\alpha^{*} \geqq 0$ such that $N_{1} \supset\left(\alpha^{*}, \infty\right)$ with $\alpha^{*} \notin N_{1}$. 
By Lemma 2.5 , it suffices to show that

$$
\lim _{\alpha \rightarrow\left(\alpha^{*}\right)^{+}} b(\alpha)=\infty .
$$

We shall prove the theorem according to $f(0)>0$ and $f(0)=0$.

If $f(0)>0$, we claim that $\alpha^{*}>0$. In fact, $u^{\prime \prime}(a, 0)=-f(0)<0$. Hence, there is an $\varepsilon>0$ such that $u(r, 0)<0$ for $r \in(a, a+\varepsilon)$. This implies $\alpha^{*}>0$. We claim that $\alpha^{*} \in P$. If $\alpha^{*} \notin P$, then $(0, \infty)=N \cup P$ implies $\alpha^{*} \in N$. Since $\alpha^{*} \in N_{1}$, we have $u\left(\tau\left(\alpha^{*}\right), \alpha^{*}\right) \geqq \gamma$. By Lemma 3.1, $\alpha^{*} \in N_{1}$, a contradiction. Therefore, $\alpha^{*} \in P$ and (3.5) follows.

If $f(0)=0$, then either $\alpha^{*}>0$ or $\alpha^{*}=0$. If $\alpha^{*}>0$, then the previous argument also works and then (3.5) holds. If $\alpha^{*}=0$ and (3.5) are false, then there are $b_{0}>a$ and $\delta>0$ such that $b(\alpha) \leqq b_{0}$ for all $\alpha \in(0, \delta)$. Since $\tau(\alpha) \in\left(0, b_{0}\right)$ for all $\alpha \in(0, \delta)$, there exists a sequence $\alpha_{k} \rightarrow 0$ such that $\tau\left(\alpha_{k}\right) \rightarrow \tau_{0} \in\left[0, b_{0}\right]$. Since $u\left(\tau\left(\alpha_{k}\right), \alpha_{k}\right)>\gamma$, we have $u\left(\tau_{0}, 0\right) \geqq \gamma$, a contradiction to $u(r, 0) \equiv 0$. Hence, (3.5) holds.

Corollary 3.4. Assume conditions $(\mathrm{H} 1) \sim(\mathrm{H} 3)$ are satisfied and $f(0)>0$. Then for any $a>0$, the equation

$$
\begin{aligned}
& u^{\prime \prime}(r)+\frac{n-1}{r} u^{\prime}(r)+f(u(r))=0 \quad \text { in }(a, \infty), \\
& u(a)=0 \quad \text { and } \quad u(r)>0 \quad \text { for } r>a,
\end{aligned}
$$

has a solution $u$ which satisfies (M).

Proof. In the proof of the previous theorem, we have $N_{1} \supset\left(\alpha^{*}, \infty\right)$ with $\alpha^{*}>0$ and $\alpha^{*} \in P$. By (3.5) and Lemma 2.4, $u\left(\cdot, \alpha^{*}\right)$ satisfies (M).

4. Nonexistence on wide annuli when $\mathbf{f}(0)<0$. In this section we shall prove that if $(\mathrm{H} 2)$ and $(\mathrm{H} 3)^{\prime}$ are satisfied, then $(2.1),(2.2)$ has no positive solution when $b-a$ is too large.

We first show that $P \neq \phi$ when $f(0)<0$.

LemMA 4.1. If $f(0)<0$, then there exists $\alpha_{*}>0$ such that $\left[0, \alpha_{*}\right) \subset P$.

Proof. We first prove $0 \in P$. In fact, $u^{\prime \prime}(a, 0)=-f(0)>0$ and $H(u(a, 0))=0>$ $H(u(r, 0))$ for $r>a$ implies $u(r, 0)>0$ for $r>a$. Hence $0 \in P$.

Next, let $u_{0}>0$ such that $f(u)<0$ in $\left[-u_{0}, u_{0}\right]$. Then there exist $\varepsilon>0$ and $\alpha_{0}>0$ such that for all $\alpha \in\left[0, \alpha_{0}\right]$, we have $|u(r, \alpha)| \leqq u_{0}$ in $[a, a+\varepsilon]$. Therefore, for all $\alpha \in\left[0, \alpha_{0}\right]$,

$$
u^{\prime \prime}(r)+\frac{n-1}{r} u^{\prime}(r)>0 \quad \text { in }[a, a+\varepsilon]
$$

which implies $u(r, \alpha)>0$ in $(a, a+\varepsilon]$.

On the other hand, if $H(u(a+\varepsilon, 0))<0$, by continuous dependence of o.d.e., there exists $\alpha_{*} \in\left(0, \alpha_{0}\right)$ such that $H(u(a+\varepsilon, \alpha))<0$ for all $\alpha \in\left(0, \alpha_{*}\right)$. Therefore, for any $\alpha \in\left(0, \alpha_{*}\right), H(u(r, \alpha))<0$ for $r>a+\varepsilon$, which implies $u(r, \alpha)>0$ for $r>a+\varepsilon$. This proves $\left(0, \alpha_{*}\right) \subset P$.

We now prove the main result of this section.

ThEOREM 4.2. Assume conditions ( $\mathrm{H} 2)$ and $(\mathrm{H} 3)^{\prime}$ are satisfied. Then there exists $b^{*}>a$ such that for any $b>b^{*},(2.1),(2.2)$ has no positive solution.

Proof. By Lemma 2.5, there exist $\alpha^{*}>0$ and $b_{0}>a$ such that $\left(\alpha^{*}, \infty\right) \subset N \cup T$ and $b(\alpha) \leqq b_{0}$ for all $\alpha \in\left(\alpha^{*}, \infty\right)$. On the other hand, by Lemma 4.1, there exists $\alpha_{*}>0$ such that $\left[0, \alpha_{*}\right) \subset P$. Therefore, it suffices to show that there exists $\tilde{b}_{0}>a$ such that

$$
b(\alpha) \leqq \tilde{b}_{0} \quad \text { for any } \alpha \in\left[\alpha_{*}, \alpha^{*}\right] \cap(N \cup T) .
$$


If (4.1) were false, then there would be a sequence $\alpha_{k} \in\left[\alpha_{*}, \alpha^{*}\right] \cap(N \cup T)$ and $\bar{\alpha} \in\left[\alpha_{*}, \alpha^{*}\right]$ such that $\alpha_{k} \rightarrow \bar{\alpha}$ and $b\left(\alpha_{k}\right) \rightarrow \infty$ as $k \rightarrow \infty$. By Lemma 2.4, $\bar{\alpha} \in P$ and $u(\cdot, \bar{\alpha})$ satisfies $(M)$. Now, (H3)'(ii) and $u(r, \bar{\alpha}) \rightarrow \tilde{u}$ as $r \rightarrow \infty$ with $f(\tilde{u})=0$ implies $H(u(r, \bar{\alpha})) \rightarrow$ $F(\tilde{u})<0$ as $r \rightarrow \infty$. Therefore, there exists $r_{0}>a$ such that $H\left(u\left(r_{0}, \bar{\alpha}\right)\right)<0$. By continuous dependence of o.d.e., we have $H\left(u\left(r_{0}, \alpha_{k}\right)\right)<0$ for $k$ sufficiently large, a contradiction to Lemma 2.2(ii). This proves (4.1).

5. Multiplicity results on wide annuli. In the previous sections we studied the existence of large (and nonexistence of) positive solutions for (2.1), (2.2) under the various assumptions of $f$. In this section we shall study the existence of "intermediate size" solutions of (2.1), (2.2) when $f$ may change signs several times and satisfies condition $\left(\mathrm{f}_{3}\right)$ in $\S 1$, i.e., $f$ satisfies the following hypothesis:

(H4) there exist $m$ successive numbers $\bar{u}_{m}>\bar{u}_{m-1}>\cdots>\bar{u}_{1}>0$, which satisfy

(i) $f\left(\bar{u}_{k}\right)=0$ for $k=1, \cdots, m$; and

(ii) $0<\max \left\{F(s): 0 \leqq s \leqq \bar{u}_{k-1}\right\}<F\left(\bar{u}_{k}\right)$ for $k=2, \cdots, m$.

Let $\gamma_{k}$ be the least number in $\left(\bar{u}_{k-1}, \bar{u}_{k}\right)$ such that

$$
\int_{\bar{u}_{k-1}}^{\gamma_{k}} f(u) d u=0,
$$

for $k=1, \cdots, m$, where $\bar{u}_{0} \equiv 0$. We first prove the following lemma.

Lemma 5.1. Assume there exists an $\bar{u}>0$ such that

$$
f(u)=0 \quad \text { for } u \geqq \bar{u} .
$$

Then we have the following conditions:

(i) if $u\left(r_{1}, \alpha\right)=\bar{u}$ and $u^{\prime}\left(r_{1}, \alpha\right) \geqq 0$ for some $r_{1}>a$, then for $r>r_{1}$,

$$
u(r, \alpha)=\bar{u}+\frac{1}{n-2} r_{1} u^{\prime}\left(r_{1}, \alpha\right)-\frac{1}{n-2} r_{1}^{n-1} u^{\prime}\left(r_{1}, \alpha\right) r^{2-n} ;
$$

(ii) let $U=\left\{\alpha \in(0, \infty): u\left(r_{1}, \alpha\right)=\bar{u}\right.$ for some $\left.r_{1}>a\right\}$; then there exists $\alpha^{*}>0$ such that $U \supseteq\left(\alpha^{*}, \infty\right)$.

Proof. (i) By (5.2), we have

$$
u^{\prime \prime}(r)+\frac{n-1}{r} u^{\prime}(r)=0
$$

as long as $u(r, \alpha) \geqq \bar{u}$. Therefore, by solving (5.4) with initial condition $u\left(r_{1}, \alpha\right)=\bar{u}$ and $u^{\prime}\left(r_{1}, \alpha\right) \geqq 0,(5.3)$ follows.

(ii) We shall prove (ii) by using the method of backward shooting. If (ii) were false, there would be a sequence $\beta_{k} \rightarrow \infty$ such that

$$
w_{k}(s)<\bar{u} \text { as long as } w_{k} \text { remain positive, }
$$

where $w_{k}(s) \equiv w\left(s, \beta_{k}\right)$. Let

$$
\nu_{k}=\inf \left\{\tilde{s} \in\left(0, s_{1}\right): w_{k}(s)>0 \text { and } w_{k}^{\prime}(s) \leqq 0 \text { in }\left(\tilde{s}, s_{1}\right)\right\} .
$$

We claim that

$$
\lim _{k \rightarrow \infty} \nu_{k}=s_{1}
$$

If (5.6) were false, there would be $\nu_{0}<s_{1}$ and a subsequence of $\nu_{k}$ (for simplicity, 
rename it $\nu_{k}$ ), such that $\nu_{k} \leqq \nu_{0}$ for all $k$. Therefore, by (2.15) and (5.5), we have

$$
\begin{aligned}
w_{k}\left(\nu_{0}\right) & =\beta_{k}\left(s_{1}-\nu_{0}\right)-\int_{\nu_{0}}^{s_{1}}\left(t-\nu_{0}\right) \rho(t) f\left(w_{k}(t)\right) \\
& \geqq \beta_{k}\left(s_{1}-\nu_{0}\right)-C,
\end{aligned}
$$

for some constant $C \geqq 0$, a contradiction to (5.5). Hence (5.6) holds.

On the other hand, by (5.5) again, we have

$$
\begin{aligned}
w^{\prime}\left(\tau_{k}\right) & =-\beta_{k}+\int_{\tau_{k}}^{s_{1}} \rho(t) f\left(w_{k}(t)\right) d t \\
& \leqq-\beta_{k}+C
\end{aligned}
$$

for some $C \geqq 0$. Therefore, $w^{\prime}\left(\tau_{k}\right)<0$ if $k$ is large enough, a contradiction to the definition of $\nu_{k}$. This proves (ii).

The (energy) functional we want to minimize is

$$
J(u)=\int_{a}^{b} r^{n-1}\left\{\frac{1}{2} u^{\prime 2}(r)-F(u(r))\right\} d r
$$

in $H_{0}^{1}((a, b))$, where $H_{0}^{1}((a, b))=\{u: u$ is absolutely continuous in $[a, b]$ with $u(a)=$ $0=u(b)$ and $\left.u, u^{\prime} \in L^{2}(a, b)\right\}$.

Since $f$ may change signs, the minimizer $u_{b}$ of $J$ is not necessarily positive. However, for fixed $a$, if $b$ is sufficiently large and (H4) is satisfied, then we can prove $u_{b}$ is positive. To make the proof more transparent, we begin with the study of two simple cases.

LEMMA 5.2. If $f$ satisfies the following:

(H5)(i) $f(0) \geqq 0$;

(ii) There exists $\bar{u}>0$ such that $f(\bar{u})=0$ and $f(u)>0$ in $(0, \bar{u})$, then, we have the following results:

(i) There exists $b^{*}>a$ such that for any $b>b^{*},(2.1),(2.2)$ has a positive solution $u_{b}$ that is also a local minimizer of $J(u)$ over $H_{0}^{1}((a, b))$. Moreover,

$$
0<u_{b}<\bar{u} \text { in }(a, b) \text {, }
$$

and

$$
-\frac{b^{n}}{n} F(\bar{u}) \leqq J\left(u_{b}\right) \leqq-\frac{b^{n}}{n} F(\bar{u})+C\left(b^{n-1}+1\right)
$$

for some positive constant $C$ which is independent of $b$.

(ii) If $f(0)>0$, then there exists a positive solution $\tilde{u}$ of (3.6), (3.7) and $\tilde{u}$ is strictly increasing in $(a, \infty)$ and $\tilde{u}(r) \rightarrow \bar{u}$ as $r \rightarrow \infty$.

Proof. We first modify the function outside $[0, \bar{u}]$ as Clément and Sweers did in [6]. Denote

$$
\begin{gathered}
f_{1}(u)=\left\{\begin{array}{cc}
0 & u \geqq \bar{u}, \\
f(u) & u \in[0, \bar{u}], \\
2 f(0)-f(-u) & \text { if } u<0,
\end{array}\right. \\
F_{1}(u)=\int_{0}^{u} f_{1}(t) d t \text { and } J_{1}(u)=\int_{a}^{b} r^{n-1}\left\{\frac{1}{2} u^{\prime 2}(r)-F_{1}(u(r))\right\} d r .
\end{gathered}
$$

It is easy to verify that

$$
F_{1}(|u|)=F_{1}(u)+2 f(0)|u| \quad \text { for } u<0 .
$$


Hence, for any $u \in H_{0}^{1}((a, b))$,

$$
J_{1}(|u|) \leqq J_{1}(u)
$$

Since $f(u)$ is bounded, the minimizer of $J_{1}(u)$ over $H_{0}^{1}((a, b))$ is achieved, say $u_{b}$, which is a solution of $(2.1),(2.2)$. By (5.11), $u_{b}$ can be chosen to be nonnegative. If $u_{b} \not \equiv 0$ in $(a, b)$, then by Lemma $2.3, u_{b}>0$ in $(a, b)$.

If $f(0)>0$, then $u_{b}>0$ in $(a, b)$. If $f(0)=0$, we want to prove that $u_{b}>0$ in $(a, b)$ if $b$ is large enough. This can be done by choosing appropriate test functions $u_{b}^{*} \in$ $H_{0}^{1}((a, b))$ as follows:

$$
u_{b}^{*}(r)= \begin{cases}(r-a) \bar{u} & \text { for } r \in[a, a+1], \\ \bar{u} & \text { for } r \in[a+1, b-1], \\ (b-r) \bar{u} & \text { for } r \in[b-1, b] .\end{cases}
$$

Then

$$
J_{1}\left(u_{b}^{*}\right) \leqq-\frac{b^{n}}{n} F(\bar{u})+C\left(b^{n-1}+1\right)
$$

for some constant $C$ which is independent of $b$. Therefore, if $b$ is large enough, then $u_{b}>0$ in $(a, b)$, and by Lemma 5.1(i), $u_{b}<\bar{u}$ in $(a, b)$. By (5.13), (5.8) follows. This proves (i).

To prove (ii), we first note that $f(0)>0$ implies there exists $\alpha_{*}>0$ such that $\left(0, \alpha_{*}\right) \subset N$ and

$$
\lim _{\alpha \rightarrow 0^{+}} b(\alpha)=a
$$

(see Lin [18]). On the other hand, by Lemma 5.1(ii), there exists $\alpha^{*}>\alpha_{*}$ such that $U \supset\left(\alpha^{*}, \infty\right)$. Therefore, for any $b>b^{*}$, there exists $\alpha(b) \in\left(0, \alpha^{*}\right]$ such that $u(\cdot, \alpha(b))$ is a minimizer of $J_{1}(u)$. Therefore, by (5.14) there exists $\bar{\alpha}>0$ and a sequence $b_{k} \rightarrow \infty$ such that $\alpha\left(b_{k}\right) \rightarrow \bar{\alpha}$ as $k \rightarrow \infty$. By Lemma $2.4, \bar{\alpha} \in P$ and $u(\cdot, \bar{\alpha})$ satisfies (M). By Lemma 5.1(i) and (H5)(ii), $u(\cdot, \bar{\alpha})$ is strictly increasing in $(a, \infty)$. This proves (ii).

Next, we treat the case $f(0)<0$.

LEMMA 5.3. If $f$ satisfies the following conditions:

(H5)'(i) $f(0) \leqq 0$;

(ii) There exist $\bar{u}>\underline{u}>0$ such that $f(\underline{u})=f(\bar{u})=0$ and $f(u)<0$ in $(0, \underline{u})$ and $f(u)>0$ in $(\underline{u}, \bar{u})$

(iii) $\int_{0}^{\bar{u}} f(u) d u>\overline{0}$,

then there exists $b^{*}>a$ such that for any $b>b^{*}$ there exists a positive solution $u_{b}$ of (2.1), (2.2) with $\left\|u_{b}\right\| \in(\gamma, \bar{u})$, where

$$
\int_{0}^{\gamma} f(u) d u=0 .
$$

Proof. If $f(0)=0$, then the arguments in Lemma 5.2 also work and give the result as in Lemma 5.2(i). Note that (H5)'(iii) implies $J_{1}\left(u_{b}^{*}\right)<0$ in (5.13) when $b$ is large enough.

If $f(0)<0$, then $(5.10)$ implies the extension $f_{1}$ in (5.9) is no longer suitable to the minimization problem. Therefore, we want to modify $f$ in a different way and use super- and subsolution methods to obtain solutions for (2.1), (2.2).

Since $f(0)<0$, we can extend $f$ into $\left(u_{0}, 0\right)$ such that

$$
f\left(u_{0}\right)=0, f(u)<0 \quad \text { in }\left(u_{0}, 0\right)
$$


and

$$
\int_{u_{0}}^{\bar{u}} f(u) d u>0 .
$$

Let $v=u-u_{0}$ and denote $v_{1}=\underline{u}-u_{0}$ and $v_{2}=\bar{u}-u_{0}$. Let

$$
g(v)=f(u) \text { in }\left[0, v_{2}\right] \text {. }
$$

Then $g(0)=g\left(v_{1}\right)=g\left(v_{2}\right)=0$,

$$
g(v)<0 \text { in }\left(0, v_{1}\right) \text { and } g(v)>0 \text { in }\left(v_{1}, v_{2}\right) \text {. }
$$

We then extend $g$ outside $\left[0, v_{2}\right]$ by making

$$
g(v)=0 \quad \text { for } v>v_{2}
$$

and

$$
g(v)=-g(-v) \text { for } v<0 .
$$

Denote

$$
G(v)=\int_{0}^{v} g(t) d t
$$

Then, as in (5.10),

$$
G(|v|)=G(v) \quad \text { for all } v<0
$$

and (5.17) can be rewritten as

$$
G\left(v_{2}\right)=\int_{0}^{v_{2}} g(t) d t=\int_{u_{0}}^{\bar{u}} f(u) d u>0 .
$$

Define

$$
\tilde{J}(v)=\int_{a}^{b} r^{n-1}\left\{\frac{1}{2} v^{\prime 2}(r)-G(v(r))\right\} d r
$$

in $H_{0}^{1}((a, b))$.

Let $v_{b}^{*}$ be defined as $u_{b}^{*}$ in (5.12), but replace $\bar{u}$ by $v_{2}$. Then

$$
\tilde{J}\left(v_{b}^{*}\right) \leqq-\frac{b^{n}}{n} G\left(v_{2}\right)+C\left(b^{n-1}+1\right)
$$

for some positive constant $C$ which is independent of $b$. Therefore, there exists $b^{*}>a$ such that for any $b>b^{*}$, the equations

$$
\begin{gathered}
v^{\prime \prime}(r)+\frac{n-1}{r} v^{\prime}(r)+g(v(r))=0 \quad \text { in }(a, b), \\
v(a)=0=v(b)
\end{gathered}
$$

have a positive solution $v_{b}$ which is also a minimizer of $\tilde{J}$ with

$$
\tilde{J}\left(v_{b}\right) \leqq-\frac{b^{n}}{n} G\left(v_{2}\right)+C\left(b^{n-1}+1\right)
$$

and

$$
\left\|v_{b}\right\| \in\left(\gamma-u_{0}, v_{2}\right)
$$


Let $\tilde{u}_{b}=u_{0}+v_{b}$; then

$$
\Delta \tilde{u}_{b}+f\left(\tilde{u}_{b}\right)=\Delta v_{b}+g\left(v_{b}\right)=0 \quad \text { in } a<|x|<b,
$$

and

$$
\tilde{u}_{b}=u_{0}<0 \text { on }|x|=a \text { and }|x|=b .
$$

Hence, $\tilde{u}_{b}$ is a subsolution of (2.1), (2.2). Since $\bar{u}$ is a supersolution and $\bar{u}>\tilde{u}_{b}$, by monotone iteration scheme (see, e.g., [11], [18]), there is a positive solution $u_{b}$ of (2.1), (2.2) and $u_{b}$ satisfies $\tilde{u}_{b}<u_{b}<\bar{u}$, which also implies $\left\|u_{b}\right\| \in(\gamma, \bar{u})$.

Now, we can prove the following multiplicity result for general case.

TheOrem 5.4. Assume condition (H4) is satisfied. Then, we have the following results:

(i) If $f(0) \geqq 0$, then there exists $b^{*}<a$ such that for any $b>b^{*},(2.1),(2.2)$ has at least $2 m-1$ positive solutions $\tilde{u}_{1}, \underline{u}_{2}, \tilde{u}_{2}, \cdots, \underline{u}_{m}, \tilde{u}_{m}$ with $\tilde{u}_{k-1}<\tilde{u}_{k}$ and $\underline{u}_{k}<\tilde{u}_{k}$ in $(a, b)$, and $\left\|\underline{u}_{k}\right\|,\left\|\tilde{u}_{k}\right\| \in\left(\gamma_{k}, \bar{u}_{k}\right)$ for $k=2, \cdots, m$.

(ii) If $f(0)<0$, then there exists $b^{*}>a$ such that for any $b>b^{*},(2.1),(2.2)$ has at least $m$ positive solutions $\tilde{u}_{1}<\cdots<\tilde{u}_{m}$ with $\left\|\tilde{u}_{k}\right\| \in\left(\gamma_{k}, \bar{u}_{k}\right)$ for $k=1, \cdots, m$.

Proof. We shall prove the theorem by induction on $m$.

For $m=1$, the results were proved in Lemmas 5.2 and 5.3 under the conditions (H5) and (H5)', respectively. The arguments used in the last two lemmas are also valid for general cases; thus the details are omitted.

We first study the case $f(0) \geqq 0$. For $j=2, \cdots, m$, denote

$$
\begin{aligned}
& f_{j}(u)= \begin{cases}f(u) & \text { for } u \in\left[0, \bar{u}_{j}\right], \\
0 & \text { for } u \in\left[\bar{u}_{j}, \infty\right), \\
2 f(0)-f(-u) & \text { for } u<0,\end{cases} \\
& F_{j}(u)=\int_{0}^{u} f_{j}(t) d t,
\end{aligned}
$$

and

$$
J_{j}(u)=\int_{a}^{b} r^{n-1}\left\{\frac{1}{2} u^{\prime 2}(r)-F_{j}(u(r))\right\} d r .
$$

It is clear that $f_{j+1}$ is an extension of $f_{j}$ and

$$
\text { if }\|u\|<\bar{u}_{j}, \quad \text { then } \bar{J}_{j+1}(u)=J_{j}(u)
$$

for $j=1, \cdots, m-1$.

Assume $m=j(\geqq 1)$ is true. Then there exists a $b_{j}^{*}>a$ such that for any $b>b_{j}^{*}$, $J_{j}(u)$ has a minimizer $\tilde{u}_{j, b}$ which is a positive solution of (2.1), (2.2) and satisfies

$$
\left\|\tilde{u}_{j, b}\right\| \in\left(\gamma_{j}, \bar{u}_{j}\right)
$$

and

$$
-\frac{b^{n}}{n} F_{j}\left(\bar{u}_{j}\right) \leqq J_{j}\left(\tilde{u}_{j, b}\right) \leqq-\frac{b^{n}}{n} F_{j}\left(\bar{u}_{j}\right)+C_{j}\left(b^{n-1}+1\right)
$$

for some positive constant $C_{j}$ that is independent of $b$.

Let $u_{j+1, b}^{*}$ be as in (5.12) with $\bar{u}$ replaced by $\bar{u}_{j+1}$. Then

$$
J_{j+1}\left(u_{j+1, b}^{*}\right) \leqq-\frac{b^{n}}{n} F_{j+1}\left(\bar{u}_{j+1}\right)+C_{j+1}\left(b^{n-1}+1\right)
$$


for some positive constant $C_{j+1}$ independent of $b$. Therefore, by (5.24) (5.27), there exists $\bar{b}_{j+1}^{*} \geqq b_{j}^{*}$, such that for any $b>\bar{b}_{j+1}^{*}$, minimizer $\tilde{u}_{j+1, b}$ of $J_{j+1}$ satisfies

$$
J_{j+1}\left(\tilde{u}_{j+1, b}\right)<J_{j}\left(\tilde{u}_{j, b}\right) .
$$

Hence, $\tilde{u}_{j+1, b} \neq \tilde{u}_{j, b}$ and $\left\|\tilde{u}_{j+1, b}\right\| \in\left(\gamma_{j+1}, \bar{u}_{j+1}\right)$.

Let

$$
N_{j+1}=\left\{\alpha \in N: \gamma_{j+1}<\|u\|<\bar{u}_{j+1}\right\} .
$$

Then $N_{j+1}$ is an open set and nonempty according to the last paragraph. Therefore, by Lemma 5.1, there exist two positive numbers $\bar{\alpha}_{j+1}>\underline{\alpha}_{j+1}$, such that $\left(\underline{\alpha}_{j+1}, \bar{\alpha}_{j+1}\right) \subset$ $N_{j+1}, \underline{\alpha}_{j+1} \notin N_{j+1}$ and $\bar{\alpha}_{j+1} \notin N_{j+1}$. By Lemma $2.4, \underline{\alpha}_{j+1}$ and $\bar{\alpha}_{j+1}$ belong to $P$. Hence,

$$
\lim _{\alpha \rightarrow\left(\underline{\alpha}_{j+1}\right)^{+}} b(\alpha)=\infty=\lim _{\alpha \rightarrow\left(\bar{\alpha}_{j+1}\right)^{-}} b(\alpha),
$$

and then both $u\left(\cdot, \underline{\alpha}_{j+1}\right)$ and $u\left(\cdot, \bar{\alpha}_{j+1}\right)$ satisfy (M). Therefore, there exists $b_{j+1}^{*} \geqq \bar{b}_{j+1}^{*}$, such that for any $b>b_{j+1}^{*},(2.1),(2.2)$ has at least two positive solutions having maximum value in $\left(\gamma_{j+1}, \bar{u}_{j+1}\right)$. Since $\bar{u}_{j+1}$ is a supersolution, there exists the maximum positive solution $\tilde{u}_{j+1}$ of $(2.1),(2.2)$ having maximum value in $\left(\gamma_{j+1}, \bar{u}_{j+1}\right)$. This proves (i).

Condition (ii) can be proved by using the arguments used in (i) and Lemma 5.3; thus details are omitted.

In the proof of last theorem, we obtain the following results for (3.6), (3.7).

Corollary 5.5. Assume condition (H4) is satisfied. Then, we have the following results:

(i) If $f(0)>0$, then there exist at least $2 m-1$ positive solutions $\tilde{u}_{1}, \underline{u}_{2}, \tilde{u}_{2}, \cdots, \tilde{u}_{m}$ of (3.6), (3.7) and $\tilde{u}_{j}(r) \rightarrow \bar{u}_{j}$ as $r \rightarrow \infty$ for $j=1, \cdots, m$.

(ii) If $f(0) \leqq 0$, then there exist at least $2 m-2$ positive solutions $\underline{\sim}_{2}, \tilde{u}_{2}, \cdots, \tilde{u}_{m}$ of (3.6), (3.7) and $\tilde{u}_{j}(r) \rightarrow \bar{u}_{j}$ as $r \rightarrow \infty$ for $j=2, \cdots, m$.

\section{REFERENCES}

[1] A. Ambrosetti AND P. Hess, Positive solutions of asymptotically linear elliptic eigenvalue problems, J. Math. Anal. Appl., 73 (1980), pp. 411-422.

[2] C. BANdle, C. V. CofFMAN, AND M. MARCus, Nonlinear elliptic problems in annular domains, J. Differential Equations, 69 (1987), pp. 322-345.

[3] C. BANDle AND M. K. KWONG, Semilinear elliptic problems in annular domains, J. Appl. Math. Phys., 40 (1989), pp. 245-257.

[4] H. Brezis AND L. Nirenberg, Positive solutions of nonlinear elliptic equations involving critical Sobolev exponents, Comm. Pure Appl. Math., 36 (1983), pp. 437-477.

[5] K. J. BROWN AND H. BudIN, On the existence of positive solutions for a class of semilinear elliptic boundary value problems, SIAM J. Math. Anal., 10 (1979), pp. 875-883.

[6] P. Clement AND G. SweErs, Existence and multiplicity results for a semilinear elliptic equation, Ann. Scuola Norm. Sup. Pisa, 14 (1987), pp. 97-121.

[7] C. V. Coffman, A nonlinear boundary value problem with many positive solutions, J. Differential Equations, 54 (1984), pp. 429-437.

[8] C. V. COFFMAN AND M. MARCUS, Existence and uniqueness results for semilinear Dirichlet problems in annuli, preprint.

[9] C. Cosner AND K. SCHMitT, A priori bounds for positive solutions of a semilinear elliptic equation, Proc. Amer. Math. Soc., 95 (1985), pp. 47-50.

[10] E. N. DANCER, Multiple fixed points of positive mappings, J. Reine Angew. Math., 352 (1986), pp. 46-66.

[11] E. N. DANCER AND K. SChMitt, On positive solutions of semilinear elliptic equations, Proc. Amer. Math. Soc., 101 (1987), pp. 445-452.

[12] D. G. DE FIGUEIREDO, On the existence of multiple ordered solutions of nonlinear eigenvalue problems, Nonlinear Anal., T. M. 7 A, 11 (1987), pp. 481-492. 
[13] X. GARAIZAR, Existence of positive radial solutions for semilinear elliptic equations in the annulus, J. Differential Equations, 70 (1987), pp. 69-92.

[14] P. HESS, On multiple positive solutions of nonlinear elliptic equations, Comm. Partial Differential Equations, 6 (1981), pp. 951-961.

[15] J. L. KAZDAN AND F. W. WARner, Remarks on some quasilinear elliptic equations, Comm. Pure Appl. Math., 28 (1975), pp. 567-597.

[16] S. S. LIN, On non-radially symmetric bifurcation in the annulus, J. Differential Equations, 80 (1989), pp. 251-279.

[17] - On the existence of positive radial solutions for nonlinear elliptic equations in annular domains, J. Differential Equations, 81 (1989), pp. 221-233.

[18] — Positive radial solutions and non-radial bifurcation for semilinear elliptic equations in annular domains, J. Differential Equations, 86 (1990), pp. 367-391.

[19] — Existence of positive non-radial solutions for nonlinear elliptic equations in annular domains, Trans. Amer. Math. Soc., to appear.

[20] Z. NeHARI, On a class of nonlinear second order differential equations, Trans. Amer. Math. Soc., 95 (1960), pp. 101-123.

[21] W.-M. NI AND NUSSBAUM, Uniqueness and non-uniqueness for positive radial solution of $\Delta u+f(u, r)=$ 0, Comm. Pure Appl. Math., 38 (1985), pp. 67-108.

[22] T. SUZUKI AND K. NAGASSAKI, Lifting of local subdifferentiations and elliptic boundary value problems on symmetric domains, I, Proc. Japan Acad., 64 (1988), pp. 1-4.

[23] - On the nonlinear eigenvalue problem $\Delta u+\lambda e^{u}=0$, Trans. Amer. Math. Soc., 309 (1988), pp. 591-608.

[24] S. H. WANG AND N. D. KAZARINOFF, On positive solutions of some nonlinear eigenvalue problems, preprint. 